\title{
MILLER FISHER SYNDROME: A CASE REPORT
}

\author{
Stela Atanasova ${ }^{1}$, Ivan Dimitrov ${ }^{2}$, Ara Kaprelyan ${ }^{1}$ \\ ${ }^{1}$ Department of Neurology and Neuroscience, Faculty of Medicine, \\ Medical University of Varna \\ ${ }^{2}$ Department of Nursing, Sliven Affiliate, Medical University of Varna
}

\begin{abstract}
Miller Fisher syndrome is a rare neurological disease that is considered to be a variant of Guillain-Barré syndrome. It is characterized by abnormal muscle coordination (ataxia), paralysis of the eye muscles (ophthalmoplegia), and absence of the tendon reflexes (areflexia). Additional symptoms include generalized muscle weakness and respiratory failure. Symptoms are usually preceded by a viral illness. This paper describes a clinical case of the syndrome in a patient who presented primarily with blurred and double vision, and gait disturbance.
\end{abstract}

Keywords: Miller Fisher syndrome (MFS), Guillain-Barré syndrome (GBS), ataxia, double vision

\section{INTRODUCTION}

Miller Fisher syndrome (MFS) is a variant of Guillain-Barré syndrome (GBS). It is characterized by symptoms of abnormal muscle coordination (ataxia), paralysis of the eye muscles (ophthalmoplegia), and absence of the tendon reflexes (areflexia). Some cases only present with total ophthalmoplegia. MFS is thought to result from an abnormal acute autoimmune reaction to a previous infection. It was suggested by some researches that the IgG anti-GQ1b antibody could be a marker for MFS.

Address for correspondence:

Stela Atanasova

Faculty of Medicine

Medical University of Varna

55 Marin Drinov St

9002 Varna

e-mail:atanasova.g.stela@gmail.com

Received: December 5, 2020

Accepted: December 23, 2020
While GBS affects 1 person in 100000 , MFS is even more uncommon, making up 1 in 100000 000. MFS may progress into GBS $(1,2)$. Unlike GBS, in MFS the neurological deficit starts from the upper body parts, continuing in a downward direction. The most common initial presenting symptom is diplopia, caused by external ophthalmoplegia (3). The progress of the disease continues for approximately 1 week after the initial neurologic symptoms, and a slow and gradual improvement follows. Symptoms often resolve, with full recovery within approximately 6 months. Ataxia and ophthalmoplegia resolve earlier, in the first 3 months after onset. The continuing areflexia, which persists in some cases, is not associated with movement disability. Typical complications of GBS such as respiratory failure and need for intensive care with ventilation are rare in MFS (4).

\section{CASE REPORT}

A 47-year-old man was admitted to the First Neurological Clinic of St. Marina University Hospital in Varna, Bulgaria. He presented with blurred 
vision that had appeared three days earlier, vertigo, disturbed speech, and paraesthesiae in the four extremities, followed by unstable gait. He had had an upper respiratory tract infection 10 days before these complaints. His only medical history was of asthma, which was well-controlled with salbutamol (inhaler). The patient denied smoking, alcohol or drug abuse, or exposure to neurotoxins.

The general physical examination of the patient was normal. Neurological examination showed limited ability to move the eyes in all directions, static and locomotor ataxia. No other abnormalities were noted.

Haematology and chemistry were normal. There was no elevation of inflammatory markers in the serum. The electrocardiogram demonstrated no pathologic findings. The computed tomography of the brain was normal. Magnetic resonance imaging (MRI) of the brain was performed, which only revealed mildly thickened mucosal tissue in the left maxillary sinus due to a chronic sinus disease. Otherwise, there were no areas of contrast enhancement or parenchymal abnormalities. Nerve conduction studies (NCSs) showed a sural-sparing pattern of sensory conduction abnormalities. Serology for HIV and syphilis was negative. Cerebrospinal fluid (CSF) assessment revealed mildly elevated protein count of $0.88 \mathrm{mg} / \mathrm{dL}$, which was in line with the diagnosis of GBS or MFS.

In the initial days of the hospitalization the condition of the patient continued to worsen with more pronounced limitation of eye movements and general discomfort. The patient was started on intravenous immunoglobulins. During the course of treatment he showed some clinical improvement. The follow-up a month after the hospitalization revealed that the patient was doing very well, having recovered almost completely. The slowest to resolve were his subjective visual disturbances, which were also the first symptoms to appear.

\section{DISCUSSION}

On admission of the patient to the clinic, several possible differential diagnoses were discussed, including CNS infections and demyelinating disease. Later, when lumbar puncture was performed and a mild albuminocytological dissociation was found, attention was focused on inflammatory diseases affecting the peripheral nervous system. Miller Fisher syndrome was accepted, taking into account the leading symptoms, and supported by the NCS. It should be noted that evidence suggests a rather wide range of neurological symptoms to be present, and a significant overlap to exist in MFS and other forms of GBS. Treatment considerations are similar, consisting mainly of IVIG and plasmapheresis. Due to the benign course in the present case, the latter was not performed. Familiarity with the rare Miller Fisher syndrome will help the clinician to establish the diagnosis in patients presenting with areflexia, ataxia, and ophthalmic symptoms, and to treat accordingly.

\section{REFERENCES}

1. Buchwald B, Bufler J, Carpo M, Heidenreich F, Pitz $\mathrm{R}$, Dudel J, et al. Combined pre- and postsynaptic action of IgG antibodies in Miller Fisher syndrome. Neurology. 2001;56(1):67-74. doi: 10.1212/ wnl.56.1.67.

2. Gabaldón Torres L, Badía Picazo C, Salas Felipe J. Role of neurophysiological studies in Miller-Fisher syndrome. Neurologia (English Edition). 2013;28(7):451-2. doi: 10.1016/j.nrleng.2012.04.020.

3. Anthony SA, Thurtell MJ, Leigh RJ. Miller Fisher syndrome mimicking ocular myasthenia gravis. Optom Vis Sci. 2012;89(12):e118-23. doi: 10.1097/ OPX.0b013e31827717c1.

4. Yepishin IV, Allison RZ, Kaminskas DA, Zagorski NM, Liow KK. Miller Fisher Syndrome: A case report highlighting heterogeneity of clinical features and focused differential diagnosis. Hawaii J Med Public Health. 2016;75(7):196-9. 